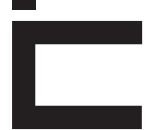

\title{
A ATUAÇÃO DO ENFERMEIRO NAS COMPLICAÇ̃̃ES PÓS-OPERATÓRIAS CARDÍACAS IMEDIATAS EM INSTITUIÇÕES HOSPITALARES DE ARACAJU-SE
}

Aline Maria Cruz Teles ${ }^{1}$

Emília Cervino Nogueira ${ }^{3}$
Débora Kelly Dias Santos Melo²

te intercorrência. Os dados foram analisados quantitativamente com auxílio do software SPSS statistic versão 20 e apresentados em forma de análise descritiva. As principais complicações pós-operatórias cardíacas imediatas são o baixo débito cardíaco, o sangramento e a hipotensão, identificadas principalmente por meio de observação do monitor multiparamétrico $(94,4 \%)$ e acompanhamento volêmico (94,4\%). As principais intervenções realizadas pelos enfermeiros para controle das intervenções são reposição volêmica $(94,4 \%)$, ordenha do dreno (83,3\%). Além da intervenção imediata, os enfermeiros realizam alguns cuidados principais como: a prevenção e tratamento da dor $(94,4 \%)$ e a prevenção e/ou controle de infecção (94,4\%). Com isso, a equipe de enfermagem é base fundamental do 
cuidado, é ela quem lida diariamente com o paciente. A atuação dos enfermeiros frente às complicações pós-operatórias cardíacas imediatas é eficaz, desde que o mesmo possua recursos materiais e humanos adequados, além de competência e habilidade, através de constantes treinamentos e capacitações.

\section{PALAVRAS-CHAVE}

Cirurgia Cardíaca. Enfermeiro. Complicações Pós-Operatórias.

\section{ABSTRACT}

Heart disease is growing in society as morbidity and mortality factor affecting the daily lives of people and quantitatively increasing surgical procedures. Therefore, this study aimed to identify the role of nurses across the immediate postoperative cardiac complications. This was an investigative study with quantitative descriptive analysis and cross during the second half of 2013 at two hospitals in Aracaju-SE approach with nurses working in the Cardiac Intensive Care Unit, or serving patients in the immediate postoperative period of cardiac surgery. A structured questionnaire with questions concerning the knowledge of postoperative complications and the performance by complication was used. Data were analyzed quantitatively with SPSS software version 20 statistic and presented in descriptive analysis. The main immediate postoperative cardiac complications are low cardiac output, bleeding and hypotension, mainly identified through observation of the multiparameter monitor (94.4\%) and blood volume monitoring (94.4\%). The main interventions performed by nurses for control of interventions are fluid replacement (94.4\%), drain milking (83.3\%). Beyond the immediate intervention, nurses perform some major attention to: the prevention and treatment of pain $(94.4 \%)$ and the prevention and / or control of infection ( $94.4 \%)$. Thus, the nursing team is cornerstone of care, it is she who deal daily with the patient. The role of nurses in the face of immediate cardiac postoperative complications is effective, provided it has adequate human and material resources, as well as competence and skills through constant training and capacity building.

\section{KEYWORDS}

Cardiac Surgery. Nurse. Postoperative Complications. 


\section{RESUMEN}

Enfermedades cardíacas han tenido un aumento tanto en la sociedad, como la morbosidad y la mortalidad que afecta la vida cotidiana de las personas y aumentan cuantitativamente los procedimientos quirúrgicos. Como objetivo fue identificar el papel de los enfermeros a través de las complicaciones cardiacas postoperatorias inmediatas. Fue un estudio de investigación con enfoque transversal y análisis descriptivo cuantitativo en el segundo semestre de 2013en dos hospital es en Aracaju-SE con las enfermeras que trabajan en la Unidad de Cuidados Intensivos Cardiaca, o que sirven a pacientes en el postoperatorio inmediato de la cirugía cardíaca. Han sido elaboradas preguntas estructuradas sobre los conocimientos acerca de las complicaciones postoperatorias y se utilizó de las actuaciones con base las complicaciones. Los datos fueron analizados cuantitativamente con la asistencia de software estadístico SPSS20y se presentan entablas y análisis descriptivo. Las principales complicaciones cardiacas postoperatorias inmediatas son bajas, como por ejemplo, la hipotensión, y hemorragia, identificado principalmente a través de la observación de Monitor multiparamétrico $(94,4 \%)$ y el seguimiento del volumen de sangre $(94,4 \%)$. Las principales intervenciones de los enfermeros para control de las intervenciones son la reposición de líquidos(94,4\%), el ordeño de la fuga(83,3\%). Además de la intervención inmediata, los enfermeros llevan algún tipo de atención importante como: la prevención y tratamiento del dolor $(94,4 \%)$ y la prevención y control de la infección (94,4\%). Así, el personal de enfermería es la base fundamental de la atención es ella la que lida a diario con el paciente. El papel de los enfermeros a través de las complicaciones cardiacas postoperatorias inmediatas es eficaz, siempre y cuando tenga los recursos humanos y materiales adecuados, así como la competencia y la habilidad a través de la capacitación constante y la creación de capacidades.

\section{PALABRAS CLAVE}

Cirugía cardiaca. Enfermero. Complicaciones Postoperatorias. 


\section{INTRODUÇÃO}

Os comportamentos de risco estão sendo disseminados atualmente, reforçando a necessidade de se discutir comportamentos saudáveis para a população. As doenças cardíacas são as principais patologias consequentes desses comportamentos, pois vêm crescendo na sociedade como fator de morbidade e mortalidade, comprometendo a vida diária das pessoas, aumentando quantitativamente os procedimentos cirúrgicos (LAIZO; DELGADO; ROCHA, 2010).

As doenças cardiovasculares representam importante problema de saúde pública não só no Brasil, mas em todo o mundo, visto que constituem a principal causa de morbimortalidade e representam os mais altos custos em assistência médica, além de elevada prevalência e incidência (LIRA et al., 2012).

Tais doenças são as principais causas de morte em mulheres e homens no Brasil e responsáveis por cerca de $20 \%$ de todas as mortes em indivíduos acima de 30 anos. Segundo o Ministério da Saúde foram 962.931 mortes em indivíduos com mais de 30 anos no ano 2009. As doenças isquêmicas do coração (DIC) foram responsáveis por 95.449 mortes e as doenças cerebrovasculares (DCbV) por 97.860 mortes (MANSUR; FARVARATO, 2012).

A cirurgia cardíaca é um advento na medicina, pois consiste em um procedimento que prolonga a vida e diminui a morbidade da doença. Os pacientes submetidos passam por uma série de exames e testes pré-operatórios, devido à sua elevada taxa de morbidade e complicações relacionadas à situação pré-operatória e à circulação extracorpórea (CEC), utilizada durante a operação, principalmente em idosos, devido ao processo inflamatório sistêmico. Por isso, os pacientes precisam ser bem preparados hemodinamicamente e psicologicamente (LAIZO; DELGADO; ROCHA, 2010).
Conforme Soares e outros autores (2011) as cirurgias cardíacas apresentam complicações típicas, sendo algumas mais prevalentes do que outras. Complicações cardíacas (infarto agudo do miocárdio e insuficiência cardíaca congestiva), hipertensão arterial pulmonar, doenças cerebrovasculares, complicações neurológicas, infecciosas e renais são frequentes.

Os mesmos autores enfatizam que os principais fatores de risco para o surgimento de alterações orgânicas no período pós-operatório de cirurgias cardíacas se associam a: idade do paciente, sexo, história médica prévia (doenças de base existentes), tipo de medicação utilizada no pré-operatório e fatores de risco intraoperatório (tipo de cirurgia, tempo de permanência em circulação extracorpórea, tempo de permanência em internação e uso de medicação específica).

O pós-operatório imediato é um período de grande relevância quando se trata de cirurgia cardíaca, pelos riscos inerentes ao próprio procedimento e visa a recuperação do paciente. Sendo assim, os cuidados de enfermagem são fundamentais (ROCHA; MAIA; SILVA, 2008).

O enfermeiro é o profissional de saúde mais presente no cuidado ao paciente no âmbito hospitalar, especialmente no período perioperatório. A relação entre paciente e enfermeiro deve ser evidenciada por retratar um vínculo importante na atenção ao sujeito, pois por meio do relacionamento proporciona tranquilidade e segurança, visando bem-estar na integração a um ambiente novo e hostil como o hospitalar. A atenção, a confiança e o apoio entre o enfermeiro e paciente constituem o fundamento do vínculo (CRUZ; LOPES, 2010).

Perante as necessidades encontradas pela enfermagem à assistência ao paciente pós-operatório cardíaco, os profissionais vêm aprimorando seus conhecimentos e propondo novas alternativas, desenvolvendo metodologia própria de trabalho, fundamentada na cientificidade. Por meio do processo de enfermagem, os profissionais têm tido a possibilidade de dar e obter respostas aos questionamentos 
referentes à qualidade do cuidado e desenvolvimento científico da enfermagem, fornecendo uma assistência sistemática e organizada ao sujeito. Sistematizar o cuidado implica em embasar cientificamente e permite a consolidação da prática de assistência do enfermeiro (TRUPPEL et al., 2009).

Portanto, diante do crescente quantitativo de doenças cardíacas na sociedade, dos elevados custos hospitalares e da indevida apresentação da enfermagem na mídia, devido à caracterização de comportamentos errôneos, percebeu-se a importância de discutir sobre o comportamento desses profissionais diante de complicações que podem provocar morbimortalidades naqueles que têm suas vidas sob seus cuidados.

Considerando a essencialidade do enfermeiro no cuidado ao paciente no perioperatório, o estudo apresentou como objetivo identificar a atuação dos enfermeiros frente às complicações pós-operatórias cardíacas imediatas.

\section{METODOLOGIA}

Tratou-se de um estudo investigativo com abordagem quantitativa transversal e análise descritiva, realizado no segundo semestre de 2013 em dois hospitais em Aracaju-SE. As duas instituições são de referência para cirurgias cardíacas, sendo uma de caráter filantrópico e a outra consiste numa instituição de cunho particular.

Foram incluídos na amostra todos os enfermeiros que atuam em Unidade de Terapia Intensiva Cardíaca, ou que atendem pacientes em pós-operatório imediato de cirurgia cardíaca, e que concordaram em participar, assinando previamente o termo de consentimento livre e esclarecido. Os enfermeiros excluídos foram aqueles que estavam de férias ou de licença ou não quiseram participar da pesquisa e/ou aqueles que não foram encontrados durante o período de coleta de dados.
Foi utilizado um questionário estruturado com questões relativas ao conhecimento sobre as complicações pós-operatórias e a atuação mediante intercorrência. 0 questionário foi elaborado pelas pesquisadoras com base em trabalhos de Araújo, Lira e Souza (2011), Bispo e outros autores (2012), Giacomazzi (2008), Gouvea (2007) e Hermida e Araújo (2006) e analisado por três juízes com vasta experiência no tema para a validação do conteúdo, sendo um médico cardiologista, um enfermeiro especialista em cardiologia e um profissional especialista em metodologia científica.

Os dados foram analisados quantitativamente com auxílio de software SPSS statistic versão 20 e apresentados em forma de tabelas e análise descritiva.

A pesquisa foi submetida à avaliação do Comitê de Ética em Pesquisa da Universidade Tiradentes de Aracau-SE, e aprovada sob o CAAE $n^{\circ}$ 20299213.8.0000.5371.

\section{RESULTADOS E DISCUSSÃO}

A população foi constituída de 21 enfermeiros, sendo onze na instituição privada e dez na filantrópica, obtendo-se uma amostra final de 18 enfermeiros $(85,7 \%)$, conforme os critérios de inclusão e exclusão.

A amostra foi caracterizada por sua maioria de jovens adultos com 55,6\% na faixa etária de 20 - 30 anos; $71,6 \%$ do gênero feminino, $50 \%$ casados e com filhos. $72,2 \%$ possuem dois vínculos empregatícios sendo $66,7 \%$ com jornada semanal de trabalho maior que 40 horas/semanais, $77,8 \%$ são pós-graduados, $55,5 \%$ possuem tempo de exercício profissional entre 1 a 6 anos, e $83,3 \%$ atuam concomitantemente em outros setores.

De acordo com Vaz e outros autores (2009) a faixa etária dos profissionais enfermeiros que trabalham em UTI é em média de 24 a 40 anos, pois percebe- 
-se que os enfermeiros mais jovens se envolvem com áreas de grande complexidade, considerando que a redução de enfermeiros atuando em UTI com mais de 40 anos pode estar relacionado ao indício de que estes profissionais são absorvidos em outros setores, procuram cargos administrativos, buscam a área de ensino ou até mesmo desistem da profissão. Entretanto, os profissionais de maior experiência têm mais possibilidades de apresentarem maior segurança técnica, e, consequentemente, chances maiores de facilidade nos controles de situações, particularmente as críticas.

Concomitante à luta por novos direitos, a categoria apresenta, também, a necessidade de sua redução de carga horária semanal de trabalho para a garantia de uma assistência com qualidade e segura, pois a necessidade de trabalhar em diversos locais para a obtenção de uma renda digna para suprir as necessidades individuais e familiares atrela ao profissional de enfermagem a exclusão ao descanso e à vida social (SANTOS et al., 2013).

Além da jornada de trabalho excessiva, foi possível identificar durante a coleta de dados a atuação em dois setores ou mais no mesmo turno de trabalho, podendo gerar uma atuação insegura, pois não permite que o profissional atue com concentração exclusiva ao setor específico, o qual é um local de grande risco e de procedimentos complexos.

Percebe-se que 66,6\% da amostra referem ser capacitadas para atuar com pacientes cardíacos, por meio de cursos $(46,2 \%)$ e congressos/seminários/jornadas e fóruns $(46,2 \%)$, destacando que na instituição privada a predominância da capacitação foi mais frequente que na filantrópica. Entretanto, observa-se que as capacitações são em sua maioria busca ativa do profissional e não provenientes de eventos organizados pela instituição trabalhada.

Diversos estudos mostram que o mercado de trabalho em unidades de grandes complexidades exige uma melhor qualificação e que o enfermeiro especializado está mais apto a prestar assistência aos pacientes críticos, facilitando assim uma maior adaptação ao setor (VAZ et al., 2009).

Quanto à capacitação do profissional, 66,7\% demonstram que os enfermeiros recebem treinamento pela instituição, principalmente quando é de cunho particular (38,9\%), entretanto a maioria não soube responder a frequência do treinamento, embora na instituição privada grande parte afirmasse ser anualmente (22,3\%). É importante ressaltar que, alguns profissionais recentemente admitidos nas instituições estudadas, podem ainda não ter recebido a capacitação, segundo o critério do calendário institucional.

A enfermagem ainda encontra dificuldades em capacitar adequadamente os profissionais para a identificação das problemáticas na assistência à saúde, especificamente no pós-operatório cardíaco (CRUZ; LOPES, 2010).

Para os autores supracitados, o número adequado de profissionais da equipe é premissa indispensável para o cuidado de qualidade e faz parte da estrutura do serviço, contribuindo para a obtenção ou manutenção de condições favoráveis no ambiente de trabalho. 0 adequado quantitativo de profissionais de enfermagem pode possibilitar menor incidência de agravos à saúde dos trabalhadores devido à redução da sobrecarga de trabalho e, consequentemente, menores riscos à saúde da clientela.

De acordo com os dados obtidos, os participantes afirmaram que as cirurgias cardíacas frequentemente realizadas são revascularização miocárdica (100\%); valvuloplastia (100\%); comunicação interatrial (94,4\%); comunicação interventricular (88,9\%) e outras $(61,1 \%)$.

Segundo Araújo, Lira e Souza (2011) existem diferentes tipos de cirurgias cardíacas, desde as minimamentes invasivas ao transplante cardíaco. As principais 
são a revascularização miocárdica, a cirurgia para valva mitral, tricúspide e para a aórtica, além de correção de aneurisma ou dissecção de aorta ascendente.

Diante dos dados coletados, foi possível identificar que as principais complicações pós-operatórias cardíacas imediatas são o baixo débito cardíaco, o sangramento e a hipotensão, contrapondo com distúrbios neurológicos, endócrinos e vasoplegia, classificadas como as complicações menos frequentes.

A hipertensão, a isquemia miocárdica, tamponamento cardíaco, fibrilação atrial, arritmias ventriculares, distúrbio de condução, dificuldades respiratórias, insuficiência renal, dor, infecção, insônia, agitação, perfusão inadequada, ansiedade, e os distúrbios gastrintestinais foram identificadas como complicações imediatas com frequências diferenciadas.

Segundo Silva e outros autores (2013), o enfermeiro precisa observar, por meio do exame físico, o paciente constantemente para a verificação do estado geral e principalmente a identificação de sangramentos nos drenos, que consiste na complicação de maior importância, devendo ser comunicada se o fluxo de sangramento for maior que $150 \mathrm{ml} / \mathrm{h}$.

Já Araújo, Lira e Souza (2011) afirmam que as principais complicações no pós-operatório cardíaco imediato podem envolver os sistemas cardiovascular, pulmonar, renal, gastrintestinal e neuropsicológico, sendo a mais comum a síndrome do baixo débito cardíaco associada comumente à pré-carga diminuída, pós-carga aumentada, arritmias, tamponamento cardíaco ou depressão miocárdica com ou sem necrose e além de sangramento excessivo resultante de coagulopatia, hipertensão ou hemostasia inadequada.

Os enfermeiros informaram utilizar os seguintes meios para identificar as complicações cardíacas imediatas: o monitor multiparamétrico (94,4\%), acompanhamento volêmico $(94,4 \%)$, exames laboratoriais e/ou de imagens $(88,9 \%)$, o exame físico $(66,7 \%)$, o processo de enfermagem (61,1\%), e o monitoramento hemodinâmico $(55,6 \%)$.

Os enfermeiros instruídos e conhecedores da realidade em que atuam têm maiores chances de contribuir com a evolução do paciente, visando a recuperação com qualidade. Ao reconhecer as complicações que podem existir, é possível desenvolver raciocínio clínico perante a situação estabelecida e realizar plano de cuidados individuais para a implementação de ações que garantam qualidade na assistência (VIVAS; FONSECA, 2010).

As intervenções realizadas pelos enfermeiros são reposição volêmica $(94,4 \%)$, ordenha do dreno $(83,3 \%)$, oxigenação $(77,8 \%)$, uso do carro de emergência $(77,8 \%)$, acionar o médico $(77,8 \%)$, monitoramento hemodinâmico $(72,2 \%)$, eletrocardiograma $(72,2 \%)$, controle rigoroso de infusões $(72,2 \%)$, manobra de ressuscitação cardíaca $(66,7 \%)$, balanço hídrico (55,6\%), administração de medicamentos prescritos (55,6\%), aspiração endotraqueal (38,9\%) e curativo compressivo $(27,8 \%)$.

A equipe de enfermagem é envolvida em todos os procedimentos no pós-operatório cardíaco imediato, desde a admissão do paciente na UTI e toda a sua assistência, tais quais: verificação dos sinais vitais (exame físico), abertura e manipulação das sondas e drenos, observação de infusão de drogas, hidratação venosa, identificação de cateteres vasculares, eletrocardiograma (ECG), balanço hídrico e observação de complicações imediatas (SILVA et al., 2013).

Os cuidados mais citados pelos enfermeiros foram a prevenção e tratamento da dor (94,4\%); a prevenção e/ou controle de infecção (94,4\%). Em segundo lugar, foram relatados: a manutenção da integridade tecidual $(88,9 \%)$ e o apoio psicológico $(88,9 \%)$. A seguir, foram citadas: a manutenção da ventilação e oxigenação $(83,3 \%)$ e a manutenção do equilíbrio hidroeletrolítico $(83,3 \%)$, além da manutenção do débito cardíaco referida por $77,8 \%$ dos enfermeiros, dentre 
outros cuidados (5,6\%), como controle de temperatura corporal, foram relatados.

Araújo, Lira e Souza (2011) corroboram com os dados encontrados ao afirmarem que os cuidados de enfermagem para o paciente em pós-operatório cardíaco imediato devem ser: a manutenção do débito cardíaco, a diminuição/ausência de dor, perfusão tissular adequada, integridade tecidual, equilíbrio hidroeletrolítico e controle da glicemia, manutenção da ventilação e oxigenação adequadas, precauções e medidas para redução de risco de infecção, comunicação, redução da ansiedade do paciente e de seus familiares atendendo às necessidades do autocuidado e fornecimento de serviço nutricional adequado.

Os principais recursos utilizados durante as complicações foram acionar o médico (100\%) e o uso do carro de emergência (94,4\%), concomitante ao uso de manta térmica $(38,9 \%)$, do soro aquecido $(33,3 \%)$ e de demais recursos $(27,8 \%)$.

Os recursos humanos identificados para ajudar no cuidado do pós-operatório cardíaco para 94,4\% dos enfermeiros foram o médico plantonista, 94,4\% também contam com a ajuda dos técnicos e auxiliares de enfermagem, $61,1 \%$ contam com o médico assistente, $61,1 \%$ com colegas enfermeiros; $55,6 \%$ contam com o médico residente e $27,8 \%$ afirmam contar com demais profissionais para a intervenção.

Segundo Bertoglio e outros autores (2008) os profissionais de enfermagem são os primeiros a responderem a situações de emergência, principalmente nos casos de parada cardiorespiratórias. A aplicação imediata, competente e segura por parte da equipe contribui para o sucesso no atendimento e consequente sobrevida. É preciso atitudes e comportamentos dos enfermeiros para que influenciem com rapidez e envolvimento a equipe de enfermagem em situações de emergência.

Quanto à organização e padronização do serviço de enfermagem para o setor de cardiologia, foi verifi- cado que $94,4 \%$ dos participantes da amostra relataram ter no setor o protocolo operação padrão.

O enfermeiro é essencial nos processos de atenção à saúde, principalmente no contexto hospitalar especificamente no perioperatório. Entretanto, para uma atuação adequada, a enfermagem deve contar com a padronização do serviço de enfermagem que possibilita a organização do cuidado por meio da sistematização, proporcionando ao enfermeiro a (re) definição de seu espaço de atuação, envolvendo demais profissionais de saúde, possibilitando a conscientização individual e grupal (CRUZ; LOPES, 2010).

As principais dificuldades encontradas pelos enfermeiros foram recursos materiais com difícil acesso (62,5\%), falta de manutenção dos equipamentos (50\%), preparo profissional inadequado (50\%), sobrecarga de atividades da unidade (50\%), falta de recursos humanos $(31,2 \%)$, e jornada de trabalho excessiva (12,5\%).

É perceptível que o profissional só pode apresentar uma boa atuação quando possui recursos materiais e humanos adequados para a assistência de qualidade. Diante de tais necessidades, a resolução COFEN 293/2004 preconiza que, para um bom dimensionamento da equipe de enfermagem, faz-se necessário considerar a instituição, o serviço de enfermagem prestado e a clientela atendida, principalmente em unidades que acolhem pacientes em estado crítico como a unidade de terapia intensiva. Neste sentido, a ANVISA estabelece em sua RDC nº 7 (BRASIL, 2010) diversos requisitos para o adequado funcionamento de uma unidade de terapia intensiva, dentre eles, o dimensionamento de um enfermeiro para cada dez leitos por turno trabalhado. Para a Associação de Medicina Intensiva Brasileira (2009), o ideal é o quantitativo de um enfermeiro assistencial para cada cinco leitos ou fração por turno.

A valorização do enfermeiro enquanto principal cuidador foi corroborado com $100 \%$ da amostra, considerando que a atuação do enfermeiro por meio da 
identificação das complicações e intervenção imediata resulta positivamente na redução da morbimortalidade dos pacientes.

A enfermagem deve participar de todo o período perioperatório cardíaco, pois é por meio do acompanhamento de sua história pregressa, evolução do tratamento, prevendo e provendo cuidados sempre que necessários que ele atua na identificação imediata de complicações que podem evoluir para morte ou aumentar o tempo de permanência hospitalar. É preciso ter sensibilidade e discernimento para saber agir de forma condizente com o período, no qual o paciente se encontra. O cuidado é essência da enfermagem (CRUZ; LOPES, 2010).

\section{CONCLUSÃO}

A equipe de enfermagem atua desde o diagnóstico até sua intervenção e por isso é base fundamental do cuidado, é ela quem lida diariamente com o paciente. A atuação dos enfermeiros frente às complicações pós-operatórias cardíacas imediatas é eficaz, desde que o mesmo possua recursos materiais e humanos adequados, além de competência e habilidade, por meio de constantes treinamentos e capacitações. Por meio do exame clínico, do olhar diferenciado é que o enfermeiro percebe o paciente e todas as suas alterações e então intervêm de forma adequada com a intercorrência.

É por meio da evolução científica que a enfermagem deve consolidar sua nova base histórica, dando continuidade a novas pesquisas para contribuir com novos conhecimentos e expondo realidades diferenciadas em cada contexto, cultura e país. A ciência deve estar inserida na sociedade e é dela que se extrai o saber.

\section{REFERÊNCIAS}

AMIB - ASSOCIAÇÃO DE MEDICINA INTENSIVA BRASILEIRA. Regulamento Técnico para funcionamento de unidades de terapia intensiva. São Paulo, 24 de abril de 2009.

ARAÚJO, W. M. de; LIRA, A. L. B. de C.; SOUZA, N. T. C. de. Mapeamento das atividades de enfermagem em pacientes submetidos à cirurgia cardíaca. 2011. $34 \mathrm{f}$. Monografia (Curso de graduação em Enfermagem), Universidade Federal do Rio Grande do Norte, Natal, 2011.

BERTOGLIO, V. M.; et al. Tempo decorrido do treinamento em parada cardiorrespiratória e o impacto no conhecimento teórico de enfermeiros. Revista Gaúcha de Enfermagem, v.29, n.3, set. 2008, p.454-60.

BISPO, G. L. R. et al. Construção e Validação do Instrumento para a consulta de enfermagem puerperal. Revista Enfermagem UFPE on line, v.6, n.3, mar. 2012, p. 596-605.

BRASIL. ANVISA - Agência Nacional de Vigilância Sanitária. Resolução de Diretoria Colegiada RDC n. 7. 24 de Fevereiro de 2010. Disponível em: <http://www. anvisa.gov.br/hotsite/segurancadopaciente/documentos/rdcs/RDC\%20N\%C2\%BA\%207-2010.pdf>. Acesso em: 20 nov. 2013.

CONSELHO FEDERAL DE ENFERMAGEM. Resolução COFEN n. 293 de 21 de Setembro de 2004. Disponível em: <http://novo.portalcofen.gov.br/resoluo-cofen-2932004_4329.html>. Acesso em: 16 nov. 2013. CRUZ, A. P. O.; LOPES, R. Diagnóstico de Enfermagem no pós-operatório de cirurgias cardíacas. Salusvita, Bauru, v.29, n.3, 2010, p.293-312.

GIACOMAZZI, L. M. Escala de acolhimento de enfermagem para o trauma. 2008. 89f. Dissertação (Programa de Pós-graduação). Universidade Federal do Paraná, Curitiba, 2008. 
GOUVEA, J. de A. Construção e validação de um instrumento de coleta de dados para recém-nascidos internados na unidade de terapia intensiva neonatal do Hospital Lauro Wanderley. 2007. 157f. Dissertação (Programa de pós-graduação em Enfermagem) Centro de Ciências da Saúde, Universidade Federal da Paraíba, João Pessoa, 2007.

HERMIDA, P. M. V.; ARAÚJO, I. E. M. Elaboração e validação do instrumento de entrevista de enfermagem. Revista Brasileira Enfermagem, v.59, n.3, maio-jun. 2006, p.314-20.

LAIZO, A.; DELGADO, E. da F.; ROCHA, G. M. Complicações que aumentam o tempo de permanência na unidade de terapia intensiva na cirurgia cardíaca. Revista Brasileira CardiovascularN v.25, n.2, 2010, p.166-171.

LIRA, A. L. B. de C. et al. Mapeamento dos cuidados de enfermagem para pacientes em pós-operatório de cirurgia cardíaca. Revista da Rede de Enfermagem do Nordeste - REVRENE, v.13, n.5, 2012, p.1171-1181.

MANSUR, A. de P.; FAVARATO, D. Mortalidade por doenças cardiovasculares no Brasil e na região metropolitana de São Paulo: Atualização 2011. Arquivo Brasileiro de Cardiololgia, v.99, n.2, 2012, p.755-761.

ROCHA, L. A. da; MAIA, T. F.; SILVA, L. de F da. Diagnósticos de enfermagem em pacientes submetidos à cirurgia cardíaca. Revista Brasileira de Enfermagem, v.59, n.3, maio-jun. 2008, p.321-326.
SANTOS, T. A. dos. et al. Significado da Regulamentação da jornada de trabalho em enfermagem. Revista enfermagem UERJ, v.21, n.2, Rio de Janeiro, abr-jun. 2013, p. 265-268.

SILVA, M. do L. N. et al. Assistência de enfermagem no pós-operatório imediato de cirurgia cardíaca. FIEP Bulletin, v.83, special edition, 2013.

SOARES, G. M. T. et al. Prevalência das principais complicações pós-operatória em cirurgias cardíacas. Revista brasileira cardiologia, v.24, n.3, mai-jun. 2011, p.139-146.

TRUPPEL, T. C. et al. Sistematização da Assistência de Enfermagem em Unidade de Terapia Intensiva. Revista Brasileira de enfermagem, v.62, n.2, mar-abr. 2009, p.221-227.

VAZ, E. M. et al. RDC 7: Conhecimento dos Enfermeiros de Unidade de Terapia Intensiva. Cadernos da Escola de Saúde, v.2, Curitiba, 2009, p.102-117.

VIVAS, J. de C.; FONSECA, R. M. Cuidados imediatos no pós-operatório cardíaco. Artigo apresentado ao curso de Especialização Lato Sensu em Enfermagem Assistência Integral à Cardiologia da Faculdade de Ciências Médicas de Minas Gerais da Fundação Educacional Lucas Machado, Belo Horizonte, 2010.
1. Enfermeira; Residente multiprofissional em saúde da família pela UFS; Pós-graduanda em enfermagem ginecológica e obstétrica pela UNIT; pós-graduanda em enfermagem do trabalho pela Anhanguera Educacional.

E-mail: alinemcteles@yahoo.com.br

2. Enfermeira; pós-graduanda em enfermagem do trabalho pela Anhanguera Educacional.E-mail: debora_kelly@yahoo.com.br

3. Enfermeira. Mestre em Ciências da Saúde. Professora da Universidade Tiradentes. E-mail: ecervino.n@ig.com.br 